\title{
Reliability of coeliac and superior mesenteric artery origin level in lumbosacral transitional vertebrae detection and vertebral numbering
}

\author{
Erhan BIYIKLI ${ }^{1}\left(\mathbb{D}\right.$, Ibrahim Halil SEVER ${ }^{2}$ (D) Feyyaz BALTACIOGLU ${ }^{3}$ \\ ${ }^{1}$ Department of Radiology, Pendik Education and Research Hospital, Marmara University, Istanbul, Turkey. \\ ${ }^{2}$ Department of Radiology, Faculty of Medicine, Demiroglu Bilim University, Istanbul, Turkey. \\ ${ }^{3}$ Department of Radiology, School of Medicine, Marmara University, Istanbul, Turkey.
}

Corresponding Author: Erhan BIYIKLI

E-mail: biyiklierhan@hotmail.com

Submitted: 23.09.2021 Accepted: 29.12.2021

\begin{abstract}
Objective: To evaluate the value of coeliac artery (CA) and superior mesenteric artery (SMA) origin levels as an anatomic marker in the detection of lumbosacral transitional vertebrae (LSTV) and vertebral enumeration.

Patients and Methods: Institutional review board approval was obtained. Routine lumbar magnetic resonance (MR) imaging that included sagittal cervicothoracic scout images in 972 cases were evaluated retrospectively. Six segments were created in the vertebral column with 7 lines. CA and SMA ostiums were localized in these segments.

Results: Coeliac artery and SMA levels were detected more caudally in lumbarized S1 and more cranially in sacralized L5 cases compared to non-LSTV cases.

Conclusion: Coeliac artery and SMA origin levels as anatomical markers are not dependable for vertebral numbering due to their wide variability.

Keywords: Lumbosacral transitional vertebrae, Coeliac artery, Superior mesenteric artery, Vertebral enumeration, Magnetic resonance imaging
\end{abstract}

\section{INTRODUCTION}

The lumbosacral transitional vertebrae (LSTV) are defined as congenital spinal anomalies consisting of sacralization of the lower lumbar vertebral segment or lumbarization of the upper sacral vertebral segment. The prevalence of LSTV in the general population was reported as between $4 \%$ and $30 \%$ [1,2].

Computerized tomography (CT) is considered to be the best imaging method for detecting LSTV due to high spatial resolution. However, CT imaging with the indication for LSTV would unnecessarily cause ionizing radiation exposure. In addition, CT is not a preferred method in assessing non-traumatic back pain. In these clinical circumstances, magnetic resonance imaging (MRI) is often preferred, given its high contrast resolution in the vertebral column and surrounding soft tissues [1].

Magnetic resonance imaging has also some downsides in vertebral enumeration and detection of LSTV, such as limitation in visualizing the thoracolumbar component, inefficacy in the identification of transitional vertebra and failure to distinguish the hypoplastic rib and the prolonged transverse process of lumbar vertebrae.

The lumbosacral transitional vertebrae identification is crucial as incorrect vertebral numbering can cause clinically irrelevant radiologic findings and surgery at the wrong level [3]. In addition, the "Bertolotti syndrome" described by Mario Bertolotti in 1917, which relates LSTV to back pain, is a controversial subject [4].

A standard method for vertebral numbering has not been established. Sagittal cervicothoracic scout images can be used if images are available. Otherwise; aortic bifurcation, right renal artery origin, conus medullaris or iliolumbar ligament level determination can be used as an anatomical indicator. However, all these techniques can lead to inaccurate results $[5,6]$.

How to cite this article: Biyikli E, Sever IH, Baltacioglu F. Reliability of coeliac and superior mesenteric artery origin level in lumbosacral transitional vertebrae detection and vertebral numbering. Marmara Med J 2022; 35(1):48-52. doi: 10.5472/marumj.1030355 
In this study, we aimed to clarify the effectiveness of the coeliac artery (CA) and superior mesenteric artery (SMA) in vertebral numbering and LSTV detection.

\section{PATIENTS and METHODS}

This study was approved by the Marmara University, School of Medicine Ethics Committee (approval number: 09.2015.354) and was performed in accordance with the ethical standards as laid down in the 1964 Declaration of Helsinki and its later amendments or comparable ethical standards.

Magnetic resonans imaging findings of 972 patients with lower back pain or lumbar radiculopathy were evaluated retrospectively. Patients with a history of serious spinal or pelvic trauma, stage 2 and over spondylolisthesis, previous infection and lumbar spinal surgery were not included in the study because they may have changed the normal anatomy significantly. Cases with significant spinal malformation and severely torturous abdominal aortic aneurysms were not evaluated.

All images were acquired with a 3.0 Tesla MR device (Verio, Siemens Healthcare, Erlangen, Germany) using dedicated lumbar spinal coil with standard lumbar MR protocol and without contrast material. All studies included T2-weighted turbo spin echo (TSE) whole spinal column counter images. For the lumbosacral vertebral imaging; sagittal and axial T1-weighted TSE (TR/TE: 380/ 9.4ms) and T2-weighted TSE (TR/TE: 3390/106) were obtained. Axial images had a matrix of $256 \times 133$ with a slice thickness of $4 \mathrm{~mm}$ and a gap of $0.4 \mathrm{~mm}$ and sagittal images had a matrix of 256x320 with a slice thickness of $4 \mathrm{~mm}$ and a gap of $0.4 \mathrm{~mm}$.

Two radiologists, with a spinal radiology experience of 10 years and 1 year, evaluated the images by consensus. Sagittal cervicothoracic counting images were used as the gold standard method for vertebral numbering. The cervicothoracic counting images of all cases were evaluated in terms of proficiency. Following this assessment, the vertebrae were numbered using the picture archiving and communications system (PACS) program with crosslink and vertebral labelling capabilities [ ] Cervical, thoracical, and lumbar vertebrae were numbered through the caudal direction from C2 (2nd cervical vertebra) level to S1 (1st sacral vertebra) level in cervicothoracic counting images; with the assumption that the cervical region carrying 7 vertebrae and the thoracic region carrying 12 vertebrae was the anatomical standard.

Sagittal T1 and T2, and axial T2 weighted images were used to assess the origin of CA and SMA. The first main vascular structure originating from the abdominal aorta was defined as the celiac truncus, and the second main vascular structure was defined as the SMA. These vascular structures were classified according to the vertebra corpus and intervertebral discs, where they were adjacent to the cross-link function. For standard assessment, the exit points from the aorta of the vascular structures were determined.

For scoring and statistical evaluation; six regions were created in the vertebral column with 7 lines passing through the middle of the T12-L1-L2 vertebra corpus and the T11-12, T12-L1, L1-L2 and L2-L3 intervertebral disc spaces. Starting from the cranial region, each region was numbered from 1 to 6 , and the CA and SMA ostiums were located accordingly.
Anatomical structures located at the boundary of two regions were given the number of the region corresponding to the majority of the diameter percentages. The minimum and maximum values, mean, median and $95 \%$ confidence intervals for each parameter were calculated using this numbering system.

With this numbering; the area between the lines passing through the T11-T12 intervertebral disc space and the middle of the T12 vertebral corpus was identified as 1 , between the middle of the T12 vertebral corpus and the T12-L1 intervertebral disc space as 2 , between the T12-L1 intervertebral disc space and the middle of the L1 vertebral corpus as 3, between the middle of the L1 vertebra corpus and the L1-L2 intervertebral disc distance as 4, between the L1-L2 intervertebral disc distance and the middle of the $\mathrm{L} 2$ vertebra corpus as 5 and between the middle of L2 vertebra corpus and the L2-L3 intervertebral disc distance as 6 (Figure 1).

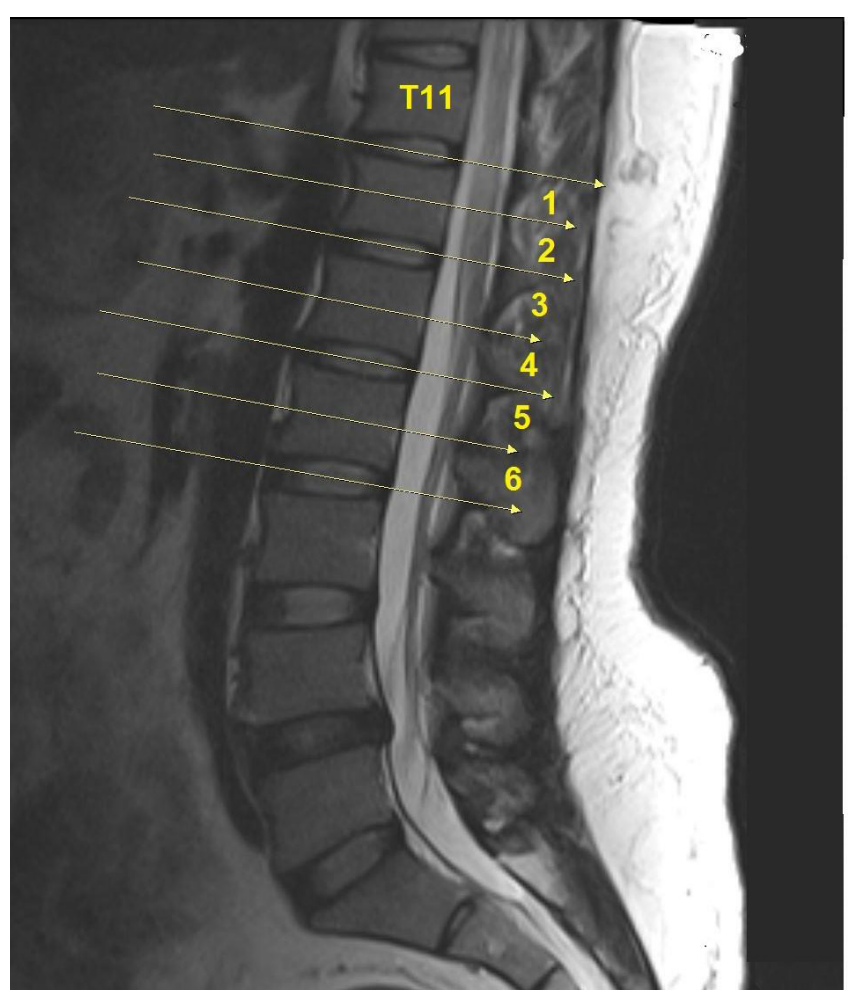

Figure 1. Sagittal T2-weighted MRI. Six artificial segments with seven lines

Finally, to evaluate the relationship between LSTV and disc degeneration, reduction of the intervertebral distance in the lumbar level was scored by numbering between 0 (none) and 5 (all levels).

\section{Statistical Analysis}

For the statistical analysis of the data obtained in the study, 'Statistical Package for Social Sciences' (SPSS, Chicago, USA) version 21.0 was used. Kolmogorov-Smirnov distribution test was used to determine descriptive statistical methods (frequency, mean, percentage, standard deviation) as well as normal distribution when study data were evaluated.

In the case of two or more groups in comparison of quantitative data; Kruskal Wallis test was used for comparison of non-normal 
distribution parameters between groups. Spearman correlation analysis was performed for the relationship of the parameters. $P$ values less than 0.05 were considered statistically significant.

\section{RESULTS}

There were 597 female (61.4\%) and 375 male (38.6\%) patients in the study $(\mathrm{F} / \mathrm{M}=1.6)$. The mean age was 43.8 .

The lumbosacral transitional vertebrae was detected in 178 (18.3\%) of the 972 cases evaluated in the study. 125 of LSTV cases had sacralized L5 and the other 53 had lumbarized S1.

In all cases; the CA ostium level was located between the 1st and 4 th regions. It was most frequently located in the second region (50.6\% of cases), followed by Region 1 (30.2\%) (Figure 2). In the non-LSTV group, the CA ostium level was mostly in the 2nd (56 $\%)$ and 1 st regions a (27\%). In lumbarized S1 cases, it was in the 3 rd ( $45 \%)$ and 2 nd regions (35\%) and in the 1 st (72\%) and 2 nd region $(28 \%)$ in sacralized L5 cases.

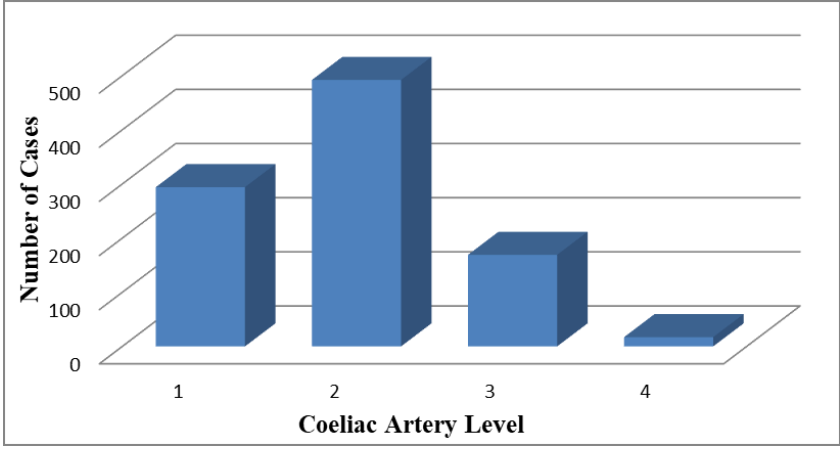

Figure 2. Distribution of CA levels

Overall SMA ostium level was observed between the 1st and 5th regions. SMA ostium level was most frequently located in the 3 rd region ( $52.5 \%$ ), followed by the 2 nd region (27.5\%) (Figure 3 ). In the non-LSTV group, it was mostly in the 3rd (59\%) and 2 nd regions (25\%), whereas in sacralized L5 cases it was in the 2nd (64\%) and 3rd regions (28\%), and in lumbarized S1 cases it was in the 4 th (49\%) and 3rd regions (35\%).

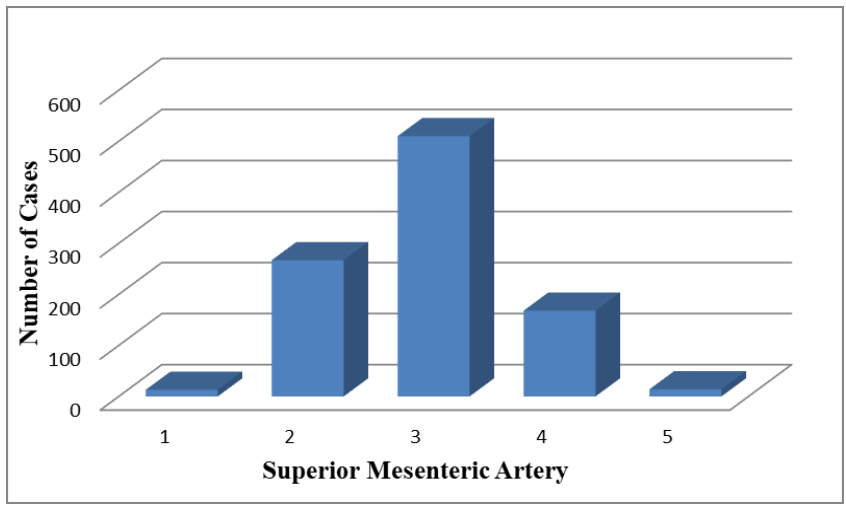

Figure 3. Distribution of SMA levels
According to these findings, CA and SMA levels showed a significant difference in distribution $(\mathrm{p}<0.001)$. In other words, CA and SMA are located higher in lumbarized S1 patients compared to non-LSTV cases. Similarly, CA and SMA of sacralized L5 cases are located lower than non-LSTV cases.

Accordingly, CA and SMA level were more caudally in lumbarized S1 and more cranially in sacralized L5 cases compared to non-LSTV cases.

Loss of disc height was not observed in $54.4 \%$ of cases, yet it was detected in $23.6 \%$ of the cases at one level, in $12.4 \%$ at two, in $5 \%$ at three, in $3 \%$ at four levels and in $1.5 \%$ at all levels. There was no significant difference in loss of disc height between the three groups (Non-LSTV, sacralized L5 and lumbarized S1) ( $\mathrm{p}$ $=0.335)$.

\section{DISCUSSION}

The results of this study showed that CA and SMA origin levels are located more caudally in the case of the lumbarization and more cranially in the sacralization, and are not dependable for vertebral numbering.

The prevalence of LSTV in the literature is reported between $4 \%$ and $37 \%$.

The systematic evaluation of some studies conducted between 2000 and 2020 found a mean LSTV prevalence of 19.4\% [618]. LSTV was detected in $18.3 \%$ of 972 cases in our study. L5 sacralization was observed in $12.9 \%$ of all cases and S1 lumbarization was observed in $5.5 \%$ of all cases. These results are in the range of current literature in terms of LSTV prevalence.

In the current literature, some studies postulate a difference in LSTV prevalence in female and male patients. In the study of Nardo et al., LSTV prevalence was found to be $11.1 \%$ in female patients and $28.1 \%$ in male patients $(\mathrm{p}<0.001)$ [17]. Similarly, in the study of Uçar et al., LSTV prevalence was found to be $17 \%$ in female patients and $20.9 \%$ in male patients $(\mathrm{p}=0.002)$ [16]. However, Tang et al. did not find a significant difference in LSTV prevalence between female and male patients [7]. Likewise, in our study, we did not find a significant difference in LSTV prevalence between female and male patients.

In a study of 534 patients with lumbar MRIs, Lee et al., reported that CA level was at the T12-L1 intervertebral disc level in $34 \%$ of the cases. They also reported that it was most commonly observed in the upper half of the L1 vertebrae in the lumbarization group and in the lower half of the T12 vertebrae in the sacralization group. There was also a statistically significant difference between these three groups $(p<0.01)$ [14]. In this study and in our study, the celiac artery was found to be lower in the lumbarized group and higher in the sacralized group compared to the normalgroup.

In our study, the CA ostium was most commonly observed in the second region (50.6\%) between the line passing through the middle of the T12 vertebra corpus and the T12-L1 intervertebral disc space, followed by the 1st region (30.2\%) which is between the line passing through the T11-T12 intervertebral disc space and the middle of the T12 vertebra corpus. In $80 \%$ of cases, the 
CA was observed at the T12 vertebral body level. CA ostium levels were significantly different between LSTV and non-LSTV cases. In lumbarized S1 cases, CA ostium levels were observed in the lower vertebral regions compared to cases without LSTV and sacralized L5. In cases of sacralized L5, it was located in the upper vertebral region compared to the non-LSTV group.

Lee et al., reported that $34 \%$ of SMA cases were observed in the upper half of the L1 vertebrae in their study. It was also reported that $73.8 \%$ of all cases were located in the T12-L1 intervertebral disc and the upper half of L1. They noted that the most frequent area where it was located was the lower half of the L1 vertebra $(47.1 \%)$ in the lumbarization group and at the level of T12-L1 intervertebral disc (35.1\%) in the sacralization group. There was also a significant difference between these three groups $(\mathrm{p}<0.01)$ [14]. In aTokgöz et al. study performed with 1049 cases, SMA was observed at the level of the L1 vertebral corpus in $55.1 \%$ of non-LSTV cases. It was noted that $58.8 \%$ of the lumbarization group was found at the L1 vertebral body level, $28.5 \%$ at the L1L2 intervertebral disc level, and $42.9 \%$ of the sacralization group at the level of the T12 vertebral corpus. A significant difference was found in the comparison of these three groups [15].

In our study, the SMA ostium was most frequently observed in the third region (52.5\%). In $27.5 \%$ of the cases, it was observed in the second region. SMA levels were significantly different in the groups with and without LSTV. SMA ostium levels in lumbarized S1 cases were observed in the lower vertebral regions compared to cases without LSTV and sacralization L5. In the case of sacralized L5, it was located in the upper vertebral region compared to the non-LSTV group.

Vascular structures can be substituted for transitional vertebrae when vertebral numbering has to be performed without whole spinal radiographs and cervicothoracic counters. A higher than expected localization of vascular structures may be a sacralization; lower localization may be an indication of the presence of lumbarization. However, the output levels of CA and SMA can not be reliably used in vertebral numbering because of their wide distribution and their differentiation by lumbarization or sacralization with LSTV presence.

To conclude, correct vertebral numbering is undoubtedly necessary to prevent errors which can occur in vertebral surgery or interventional procedures. Implementing anatomical indicators for this purpose does not seem to be efficient. In the light of our findings, we recommend obtaining whole spinal graphs or sagittal counter images before procedures and interventions that require vertebral numbering.

\section{Compliance with Ethical Standards}

Ethical Approval: This study was approved by the Marmara University School of Medicine Ethics Committee (approval number: 09.2015.354).

Financial Support: The authors have no relevant financial information to disclose.

Conflict of Interest: The authors have no potential conflicts to declare.
Authors' Contributions: EB and FB: Conceptualization, EB and IHS: Data collection, EB and FB: Data analysis, EB and FB: Investigation, FB: Supervision, EB, IHS and FB: Writing original draft, EB and IHS: Writing - Review and editing. All authors read and approved the final version of the article.

\section{REFERENCES}

[1] Konin GP, Walz DM. Lumbosacral transitional vertebrae: Classification, imaging findings, and clinical relevance. Am J Neuroradiol 2010; 31:1778-86. doi.10.3174/ajnr.A2036.

[2] Tini PG, Wieser C, Zinn WM. The transitional vertebra of the lumbosacral spine: its radiological classification, incidence, prevalence, and clinical significance. Rheumatology 1977; 16:180-5. doi:10.1093/rheumatology/16.3.180.

[3] Carrino JA, Campbell PD, Lin DC, et al. Effect of spinal segment variants on numbering vertebral levels at lumbar MR imaging. Radiology 2011; 259:196-202. doi: 10.1148/ radiol.11081511.

[4] Jancuska JM, Spivak JM, Bendo JA. A review of symptomatic lumbosacral transitional vertebrae: Bertolotti's syndrome. Int J Spine Surg 2015; 9:1-18. doi:10.14444/2042.

[5] Lee CH, Seo BK, Choi YC, et al. Using MRI to evaluate anatomic significance of aortic bifurcation, right renal artery, and conus medullaris when locating lumbar vertebral segments. Am J Roentgenol 2004 ;182:1295-300. doi: 10.2214/ ajr.182.5.1821295.

[6] Tureli D, Ekinci G, Baltacioglu F. Is any landmark reliable in vertebral enumeration? A study of 3.0-Tesla lumbar MRI comparing skeletal, neural, and vascular markers. Clin Imaging 2014 ;38:792-6. doi: 10.1016/j.clinimag.2014.05.001.

[7] Tang M, Yang XF, Yang SW, et al. Lumbosacral transitional vertebra in a population-based study of 5860 individuals: prevalence and relationship to low back pain. Eur J Radiol 2014; 83:1679-82. doi: 10.1016/j.ejrad.2014.05.036.

[8] Erken E, Ozer HTE, Gulek B, Durgun B. The association between cervical rib and sacralization. Spine (Phila Pa 1976) 2002; 27:1659-64. doi:10.1097/00007.632.200208010-00013.

[9] Apazidis A, Ricart PA, Diefenbach CM, Spivak JM. The prevalence of transitional vertebrae in the lumbar spine. Spine J 2011 ;11:858-62. doi: 10.1016/j.spinee.2011.08.005.

[10] French HD, Somasundaram AJ, Schaefer NR, Laherty RW. Lumbosacral transitional vertebrae and its prevalence in the Australian population. Glob Spine J 2014; 4:229-32. doi:10.1055/s-0034.138.7808

[11] Hsieh CYJ, Vanderford JD, Moreau SR, Prong T. Lumbosacral transitional segments: classification, prevalence, and effect on disk height. J Manipulative Physiol Ther 2000; 23:483-9. doi:10.1067/mmt.2000.108817.

[12] Delport EG, Cucuzzella TR, Kim N, Marley JK, Pruitt C, Delport AG. Lumbosacral transitional vertebrae: incidence in a consecutive patient series. Pain Physician 2006 ;9:53-6.

[13] Luoma K, Vehmas T, Raininko R, Luukkonen R, Riihimäki H. Lumbosacral transitional vertebra: relation to disc degeneration and low back pain. Spine (Phila Pa 1976) 2004; 29:200-5. doi: 10.1097/01.BRS.000.010.7223.02346.A8. 
[14] Chang HL, Park CM, Kyeong AK, et al. Identification and prediction of transitional vertebrae on imaging studies: anatomical significance of paraspinal structures. Clin Anat 2007; 20:905-14. doi:10.1002/ca.20540.

[15] Tokgoz N, Ucar M, Erdogan AB, Kilic K, Ozcan C. Are spinal or paraspinal anatomic markers helpful for vertebral numbering and diagnosing lumbosacral transitional vertebrae? Korean J Radiol 2014; 15:258-66. doi: 10.3348/kjr.2014.15.2.258.

[16] Ucar D, Ucar BY, Cosar Y, et al. Retrospective cohort study of the prevalence of lumbosacral transitional vertebra in a wide and well-represented population. Arthritis 2013; 2013:1-5. doi: 10.1155/2013/461425.

[17] Nardo L, Alizai H, Virayavanich W, et al. Lumbosacral transitional vertebrae: association with low back pain. Radiology 2012; 265:497-503 doi: 10.1148/radiol.12112747.

[18] Hanhivaara J, Määttä JH, Niinimäki J, Nevalainen MT. Lumbosacral transitional vertebrae are associated with lumbar degeneration: retrospective evaluation of 3855 consecutive abdominal CT scans. Eur Radiol 2020; 30:3409-16. doi: $10.1007 / \mathrm{s} 00330.020 .06691-2$. 